\title{
Grain flow as a fluid-mechanical phenomenon
}

\author{
By P. K. HAFF \\ Division of Physics, Mathematics and Astronomy, California Institute of Technology, \\ Pasadena, California 91125
}

(Received 26 February 1982 and in revised form 7 April 1983)

The behaviour of granular material in motion is studied from a continuum point of view. Insofar as possible, individual grains are treated as the 'molecules' of a granular 'fluid'. Besides the obvious contrast in shape, size and mass, a key difference between true molecules and grains is that collisions of the latter are inevitably inelastic. This, together with the fact that the fluctuation velocity may be comparable to the flow velocity, necessitates explicit incorporation of the energy equation, in addition to the continuity and momentum equations, into the theoretical description. Simple 'microscopic' kinetic models are invoked for deriving expressions for the 'coefficients ' of viscosity, thermal diffusivity and energy absorption due to collisions. The 'coefficients' are not constants, but are functions of the local state of the medium, and therefore depend on the local 'temperature' and density. In general the resulting equations are nonlinear and coupled. However, in the limit $s \ll d$, where $s$ is the mean separation between neighbouring grain surfaces and $d$ is a grain diameter, the above equations become linear and can be solved analytically. An important dependent variable, in this formulation, in addition to the flow velocity $\boldsymbol{u}$, is the mean random fluctuation ('thermal') velocity $\bar{v}$ of an individual grain. With a sufficient flux of energy supplied to the system through the boundaries of the container, $\bar{v}$ can remain non-zero even in the absence of flow. The existence of a non-uniform $\bar{v}$ is the means by which energy can be 'conducted' from one part of the system to another. Because grain collisions are inelastic, there is a natural (damping) lengthscale, governed by the value of $d$, which strongly influences the functional dependence of $\bar{v}$ on position. Several illustrative examples of static $(\boldsymbol{u}=0)$ systems are solved. As an example of grain flow, various Couette-type problems are solved analytically. The pressure, shear stress, and 'thermal' velocity function $\bar{v}$ are all determined by the relative plate velocity $U$ (and the boundary conditions). If $\bar{v}$ is set equal to zero at both plates, the pressure and stress are both proportional to $U^{2}$, i.e. the fluid is non-Newtonian. However, if sufficient energy is supplied externally through the walls $(\bar{v} \neq 0$ there), then the forces become proportional to the first power of $U$. Some examples of Couette flow are given which emphasize the large effect on the grain system properties of even a tiny amount of inelasticity in grain-grain collisions. From these calculations it is suggested that, for the case of Couette flow, the flow of sand is supersonic over most of the region between the confining plates.

\section{Introduction}

It is the purpose of this paper to outline a theory of grain flow which is based upon the description of continuous matter fields as usually encountered in fluid mechanics. Until fairly recently the topic of grain flow as a subject of theoretical attention has languished in a state of inattention from physicists and fluid mechanicians. Despite 
the ubiquitous appearance of granular matter in industrial, agricultural and geological contexts, and the concomitant need to transport, control and understand it, it is only within recent years that theoretical work has begun to progress beyond the pioneering investigations of Bagnold $(1954,1956)$. Many of the investigations which have been carried out to date have dealt with the general form of the flow equations (Cowin 1978; Oshima 1978; Ackermann \& Shen 1978; Savage 1979), while others have turned attention to an elucidation of the physical phenomena that underlie, at the level of the individual grain, the overall flow patterns (Ogawa 1978; McTigue 1978; Ogawa, Umemura \& Oshima 1980).

The recent contribution of Savage \& Jeffrey (1981), which was brought to the attention of the writer after the present work was completed, is in the latter category. These authors adopt a molecular picture of grain motion which has much in common with the point of view taken here. Their approach, however, is to a large extent complementary to ours. In this paper we have attempted to write down a set of complete, if heuristic, equations, which are modelled insofar as possible on the usual equations of hydrodynamics. The equations are heuristic in that they are not derived in a rigorous sense from the underlying particle interactions. Rather, the appropriate conservation laws are expressed in terms of macroscopic variables judged to be suitable, and, where required, an appeal to a very definite picture of grain-grain interactions is made in order to specify completely the various constitutive relations. In the paper of Savage \& Jeffrey, the stress tensor is calculated by appeal to particle distribution functions, so that a more definite link to the microscopic nature of the fluid is achieved. However, an explicit treatment of the energy equation is not attempted by them, with the result that solutions for the flow require additional assumptions which are not necessary in the present work.

Jenkins \& Savage (1981) have stressed the importance of the fluctuation or 'thermal' velocity in any complete model of rapid shear flow, and, using analogies with fluid turbulence, have formulated the boundary-value problem for rapidly sheared granular materials, although they did not solve their model. In the work presented here, a complete model is formulated by direct appeal to the nature of grain-grain collisions, and explicit solutions for simple flow geometries are obtained. This formulation, like that of Jenkins \& Savage (1981) avoids the difficulties of the original Bagnold model, which, as pointed out by Jenkins \& Cowin (1979), fails for certain shear flows. (In particular, the present model predicts a uniform horizontal stress in vertical channel flow.)

In the work of Ogawa (1978) the explicit incorporation of the equation governing the thermal velocity was advocated. Ogawa et al. (1980) applied this idea to the development of constitutive equations and solved several boundary-value problems in a simple kinematic model. However, their formulation of the flow problem was incomplete in the sense that some flow field variables (e.g. the density) were not explicitly determined. In the present work, all such flow-field variables are determined self-consistently; the model has no adjustable parameters, but depends only upon quantities related to the nature of the grain-grain interaction. $\dagger$

In the work reported here, once the equations have been written down they are solved essentially exactly and analytically. Although this typically requires a flow geometry or circumstance which is inconvenient or unrealistic for practical experi-

+ After this paper had been submitted for publication the works of Ackermann \& Shen (1982) and Shen \& Ackermann (1982) appeared in print. These authors developed a constitutive relation which is free of arbitrary parameters. but did not attempt a self-consistent solution of the flow equations. 
mental purposes, it is felt that the insights obtainable from exact analytical solutions are worth this price. In the future it may turn out that pieces of the theoretical picture proposed here require modification, but we believe that the general nature of the approach described below has much to recommend it. Not of least importance is the interest attaching to a consistent treatment of self-exciting systems of the type often exemplified by grain flows. The equations and solutions below represent a definite and consistent description of a model containing many of the characteristics one would expect in a typical grain system, and the study and explication of these equations and solutions is therefore useful and interesting.

\section{Some comparisons between granular systems and simple fluids}

Before continuing on with the matter of writing down possible flow equations, it is worthwhile listing some of the ways in which granular systems can be compared and contrasted with simple fluid systems.

\subsection{Grain size vs. molecule size}

As far as the constituent particles are concerned, the most obvious difference between grains and molecules lies in their size (or mass). A sand grain, which we take as an archetypal grain particle, is of the order of $10^{18}$ times more massive and voluminous than say a water molecule. This difference, although striking, is not a particularly fundamental one as far as the microscopic description of particle motions are concerned-both can be treated according to the laws of classical mechanics. However, grain size has an important bearing on the applicability of the continuum hypothesis as discussed below.

\subsection{Conservation of energy}

Although the trajectories of both grains and molecules can be described by classical mechanics, the quantum-mechanical nature of the molecule is exhibited by its ability to undergo completely elastic collisions. Grains, however, are totally classical, and every collision always involves a loss of kinetic energy which appears as true heat in the grains which collide. In granular systems, the fact that grain-grain collisions do not conserve kinetic energy leads to a strikingly different behaviour of the macroscopic system from what would be expected for a molecular fluid. This is true even if the inelastic loss per collision is very small.

\subsection{Grains are not identical particles}

Unlike molecules, grains are not identical particles : no two grains look precisely alike. It is to be expected that the existence of an essentially continuous spectrum of grain sizes in a given granular system would introduce significant complications into the effort to find a succinct theoretical description of that system. In the work reported here we specialize to grain systems where all particles are roughly the same size. It is not expected that small differences in size will have large effects on the motion, other factors remaining the same.

\subsection{Grain-grain interactions are noncentral}

Because real grains are not exactly spherical - and some are very far from spherical and because the surfaces of grains are typically rough, so that frictional (tangential) forces can exist, the grain-grain force is not central. This means that in most grain-grain collisions mutual torques are exerted, and therefore grain rotation, or 
spin, must accompany the motion of any real granular system. Although molecules are not necessarily spherical, many are reasonably round, and in the molecular case there is no analog to the macroscopic friction force. In this paper we assume the grain to be at least approximately spherical, and we neglect the effect of grain spin. Rotation is included in the computer-simulation calculations of Campbell \& Brennen (1982) and the inclusion of grain rotation at a formal level has been addressed by Oshima (1978), but no analytic solutions that include rotation seem to be available.

\subsection{The validity of the continuum hypothesis}

Because of the size of the grain particles, it follows that their number density is much smaller than the number density of particles in the corresponding molecular fluid. This raises some doubts about the validity of the usual continuum hypothesis. A cubic $\mathrm{mm}$ of water contains about $10^{19}$ molecules, but the same volume of sand might contain only $\sim 10$ grains or less. In a hydrodynamic system, some macroscopic quantity, such as the flow velocity, could change significantly over a distance of $1 \mathrm{~mm}$, but the number of molecules involved is so huge that we can imagine dividing up $1 \mathrm{~mm}^{3}$ into still smaller volumes, each containing many molecules, across whose linear dimensions the change in flow velocity is very small. However, if a quantity like flow velocity changes rapidly over $1 \mathrm{~mm}$ in the case of a sand-grain system, it is no longer clear that the continuum picture applies.

If the only lengthscale in an experiment involving granular flow were the grain size itself, then we would expect the continuum hypothesis to apply equally well to hydrodynamical systems and to granular matter (because there would be essentially no way to distinguish between the two systems). However, there are at least two further, independent, lengths that always enter any grain flow experiment. One of these is the size of the container confining the system. The ratio of this linear dimension to the molecular diameter might be on the order of $10^{8}$, while the corresponding ratio would be only $10^{3}$ for a typical sand-grain system (and sand grains are very small compared with many granular particles of interest). For this reason, in any real experiment, granular systems are always 'lumpy' in a sense which can never be removed by scaling.

Another lengthscale, which we shall investigate in detail below, arises from the inevitable existence of inelasticity in grain-grain collisions. To see this, it is useful to consider the response of the system to a localized input of energy. After a certain average number of collisions $n$, the total kinetic energy in the pulse will be degraded by a factor $\mathrm{e}^{-1}$. The corresponding radius of the pulse at that time provides the desired lengthscale $l_{\mathrm{i}}$. If the inelasticity is not small, $n$ will not be large, and therefore $l_{\mathrm{i}}$ will be only a few multiples of the grain diameter. + Thus, at least as far as energy transport is concerned, it is not difficult to conceive of situations where substantial changes in macroscopic quantities can occur over distances measured in small numbers of grain diameters. Making the best of this situation, we specialize to those situations where the continuum hypothesis is not obviously violated, and in those situations we assume that it is valid.

\subsection{Grain-grain interactions have no long-range attraction}

Typically the molecule-molecule force in a fluid has a repulsive core due to the exclusion principle, and a weak but relatively long-range attractive piece which is

† In the present paper we consider only systems where the grains are close together, i.e. where the average separation of the centres of adjacent grains is only slightly greater than a diameter. 


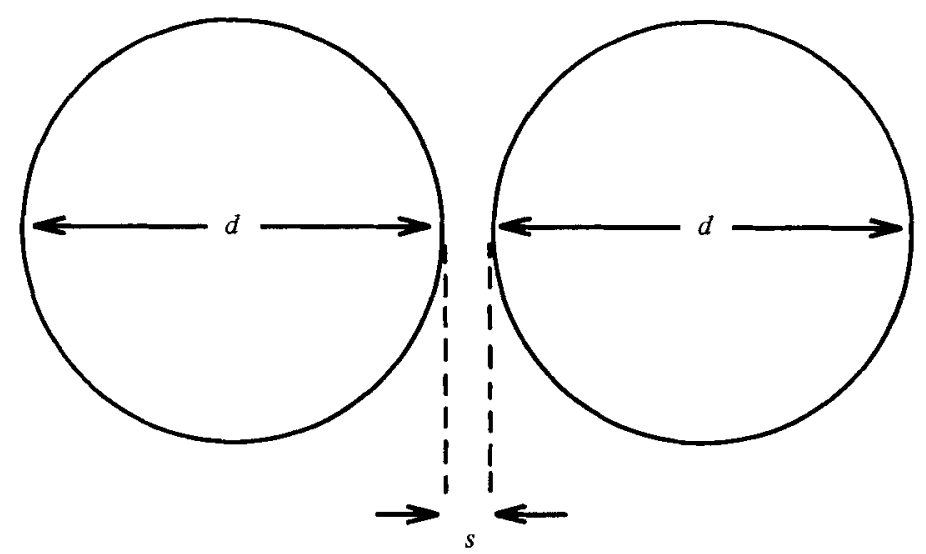

Figure 1. The average separation of neighbouring grains, diameter $d$, is designated as $s$.

responsible for phenomena like surface tension, and which figures importantly in the evaluation of fluid parameters such as the viscosity. In the grain systems that we consider in this paper, the (elastic part of the) grain-grain interaction is taken to be infinite, as in hard-sphere scattering, and there is assumed to be no attractive part. It is imagined that in the flow of dry sand, or of similar material, this ideal interaction picture is closely approached. If electrical charging effects are deemed to be important in particular cases, or if surface films present on the grains cause a certain amount of cohesion, then the approximations and assumptions adopted here will have to be revised accordingly.

\subsection{The binary collision hypothesis}

A simplifying assumption frequently made for molecular fluids, though of doubtful validity for non-dilute systems, is that only pairwise collisions are important in the dynamical evolution of the fluid. This assumption certainly breaks down in principle at densities where the tails of the molecular potentials of nearest neighbours in the fluid begin to overlap substantially. However, in a true hard-sphere fluid, because of the lack of such potential tails, the binary collision hypothesis will remain valid at essentially all densities. (In the granular case, the range $\sigma$ of the surface interaction is of course not zero, but is determined by features such as small-scale surface roughness; however, the ratio of interaction-range to grain size $\sigma / d \ll 1$. For molecules, the equivalent ratio is on the order of unity.) This hypothesis, therefore, may be a much better one for granular systems of the type to be described here than for molecular fluid systems.

\section{The physical state of the medium}

We have already referred to certain assumptions about the physical state of the particles making up the granular systems to be discussed here, i.e. the particles are assumed to be spherical, or nearly so, to be without cohesion, and so forth. In addition to these requirements, we will restrict ourselves to bulk mass densities $\rho$ that are large enough that the average spacing between nearest neighbours is always less than the grain diameter $d$. If $s$ is the average separation distance of grain surfaces between nearest neighbours, as illustrated in figure 1 , then it is always assumed that

$$
s \ll d \text {. }
$$


For many grain flows, this condition is probably fairly well obeyed over a large fraction of the flow volume. However, it will develop that it is easy to generate solutions to the equations of motion which violate (3.1), and which are therefore inconsistent with the conditions used for setting up the equations in the first place. All solutions must therefore be checked against (3.1) before being accepted.

When (3.1) is true, there is a simplification in the equations of motion which results from the fact that the density $\rho$ is nearly constant. The dependence of $\rho$ on $s$ is

so that

$$
\rho \sim \frac{m}{(s+d)^{3}}
$$

$$
\rho \sim \frac{m}{d^{3}}
$$

under condition (3.1). This has the practical effect that derivatives of the density can usually be put equal to zero, with a concomitant reduction in the complexity of the equations.

On the other hand, we anticipate that the transport process will typically involve a collision rate proportional to $s^{-1}$. Since $s^{-1}$ can range over most of the interval $\left[d^{-1}\right.$, $\infty]$ and still satisfy (3.1), it is clear that the transport properties are very sensitive to $s$ and therefore to the density; i.e. although the density is nearly constant, so that there is little variation in inertial terms due to changes in $\rho$, the very small changes in density that do occur are critical for describing the transport of various physical quantities, and this dependence on density must be retained.

It must also be pointed out that (3.2) is true only in some average sense. That is, there need not be a one-to-one relation between the spacing parameter $s$ and the density $\rho$. For high densities, the mode of grain packing will influence the density. Thus, in principle, $\rho$ might be written as a function both of $s$ and a (local) coordination number $\nu$. The developments in this paper are predicated upon the assumption that (3.2) can be written as an equality with a constant of proportionality calculated from some effective value $\bar{v}$ of $\nu$. If this is true, the precise value of $\bar{v}$ is unimportant because we attempt here only to illustrate the general trends and dependences of the theory. However, it is necessary to be aware that in proceeding along this path some part of the dynamics may be lost. For example, under suitable conditions the system might be able to perform oscillations between coordination states, at fixed $s$. In light of the complexity of the flow equations, as discussed below, it seems to be necessary at present to adopt the assumption of essentially constant $v$, and we do so henceforth.

Another qualification is that although we require $\rho$ to be large so that $s \ll d$, we do not allow the possibility $s=0$. In any given collision, of course, the separation between the colliding grains goes to zero, but $s$ defined at that point nevertheless remains non-zero since it is the average over a number of grains in the vicinity of that point. If in fact $s \rightarrow 0$ in some portion of the flow volume, then the rheology of the flow may be different from that which is described in this paper. For $s=0$ the grains would in general not lose contact for extended periods of time, but would scrape and slide over one another. For $s \neq 0$, the grains continually bounce against one another like molecules in a fluid. It is the latter circumstance treated here. Clearly there are situations where $s=0$; this is the case in a pile of grains at rest, for instance. But with sufficiently energetic flow (high shear rate), it can be anticipated that a rheology describable in terms of grain-grain collisions will be realized (Ogawa 1978; MeTigue 1978; Ogawa et al. 1980; Savage \& Jeffrey 1981).

A final remark is required about the role of interstitial fluid. The results of this 
paper are all based upon the assumption that the grain trajectories between collisions are unaffected by the presence of air, water or other fluid. Strictly speaking then the present calculations apply to grain flow in a vacuum. However, the effects of an interstitial fluid are expected to be small if the effective viscosity due to grain-grain collisions, as described in $\$ 5.2$, is substantially larger than the viscosity of the intergranular fluid. For sufficiently dense grains, this condition seems to be met if the fluid filling the pores is air. (We may note, conversely, that momentum transport by grain collisions may play a role in the behaviour of fluidized beds (Marble 1964).)

With these assumptions it is now possible to proceed directly to the equations describing grain flow.

\section{The equations of motion}

\subsection{The continuity equation}

If $\boldsymbol{u}=\left(u_{1}, u_{2}, u_{3}\right)$ is the macroscopic flow velocity of the grain system, then conservation of mass leads, as in fluid mechanics, to

$$
\frac{\partial \rho}{\partial t}+\frac{\partial}{\partial x_{i}}\left(\rho u_{i}\right)=0
$$

where $\boldsymbol{x}=\left(x_{1}, x_{2}, x_{3}\right)$ is the position coordinate. Repeated indices are summed. Since we have taken $\rho \approx$ constant, the above equation leads to

$$
\boldsymbol{\nabla} \cdot \boldsymbol{u}=0 .
$$

\subsection{The momentum equation}

We wish to write down an equation describing the conservation of momentum for a granular system. In hydrodynamics, the momentum equation (Navier-Stokes equation) incorporates a viscous force term which is proportional to $\partial u_{i} / \partial x_{k}$. The proportionality coefficient $\eta$, the dynamic viscosity, can be dimensionally composed of a density, a length and a velocity. In hydrodynamics, that velocity is very nearly the thermal velocity, which to a good approximation is constant, and therefore in many cases $\eta$ can be taken to be a constant parameter. In granular flow, a similar viscous term can be constructed dimensionally, but since the only velocity in the problem is often just the flow velocity, the viscous force term is nonlinear in $u$, and hence the flow must be non-Newtonian. This approach has been used by others to generate a momentum equation (Bagnold 1954; McTigue 1978).

The approach taken here differs slightly in philosophy. Since flow velocities are supposed to vary slowly from point to point in the granular system, and since viscosity arises from the relative motion of different parts of the system, there is no reason at this stage to suppose that the viscous force term differs in form from the corresponding result for a true hydrodynamic system. Accordingly we take the momentum equation to be given by the Navier-Stokes form

$$
\frac{\partial}{\partial t}\left(\rho u_{i}\right)=-\frac{\partial}{\partial x_{k}}\left[p \delta_{i k}+\rho u_{i} u_{k}-\eta\left(\frac{\partial u_{i}}{\partial x_{k}}+\frac{\partial u_{k}}{\partial x_{i}}\right)\right]+\rho g_{i},
$$

where $p=p(x, t)$ is the pressure and $g_{i}$ is a component of the gravitational acceleration $g$. In writing (4.3) we have made use of (3.1) in order to eliminate the term involving second viscosity.

The quantity $\eta$ can be called a viscosity coefficient, but it plays a more active role here than it does in hydrodynamics. The transport properties of the medium depend 
upon the relative velocity of neighbouring grains. Although in general this velocity must scale with $|\boldsymbol{u}|$, it need not have the directional property of the flow velocity. 'Therefore, as the simplest assumption, we take $\eta$, and other similar 'coefficients', to depend upon the energy of the flow. It follows that a consistent description of grain flow systems must necessarily involve the energy equation, in addition to (4.1) and $(4.3)$.

\subsection{The energy equation}

The conservation of energy, like conservation of momentum, is taken here to be essentially of the hydrodynamic form,

$$
\begin{aligned}
\frac{\partial}{\partial t}\left(\frac{1}{2} \rho u^{2}+\frac{1}{2} \rho \bar{v}^{2}\right)= & -\frac{\partial}{\partial x_{k}}\left[\rho u_{k}\left(\frac{p}{\rho}+\frac{1}{2} u^{2}+\frac{1}{2} \bar{v}^{2}\right)-u_{i} \eta\left(\frac{\partial u_{i}}{\partial x_{k}}+\frac{\partial u_{k}}{\partial x_{i}}\right)-K \frac{\partial}{\partial x_{k}} \frac{1}{2} \rho \bar{v}^{2}\right] \\
& +\rho u_{i} g_{i}-I .
\end{aligned}
$$

The introduction of the energy equation into the description of granular flow has been attempted by Ogawa (1978) and Ogawa et al. (1980), and the presence of a fluctuating velocity component has been discussed by Jenkins \& Cowin (1979). The velocity $\bar{v}$ is the analog in the granular system of a thermal velocity. Accordingly, $K$ functions like a thermal diffusivity. (However, like $\eta$, its form and values will be determined by the state of flow, as discussed below.) Since we have partitioned the energy into two parts, overall flow kinetic energy $\frac{1}{2} \rho u^{2}$ and internal energy $\frac{1}{2} \rho \vec{v}^{2}$, it is not surprising that $\frac{1}{2} \rho \bar{v}^{2}$ should behave much like a 'temperature' of the system. This parallel provides a convenient and expressive way of discussing the behaviour and attributes of such systems. We shall use this language, but caution that there is no implication that a complete thermodynamic analogy pertains. For example, the grain system is usually far from equilibrium, and it is not known whether the velocity distribution function, at a point where the internal (translational) energy per particle is $\frac{1}{2} m \vec{v}^{2}$, is in fact characterized by a Maxwell-Boltzmann form with ' $k T$ ' corresponding to $\frac{1}{2} m \bar{v}^{2}$. (Note that Savage \& Jeffrey (1981) assume a Maxwellian distribution. $\dagger$ )

The quantity $I$ gives the rate at which energy is lost from the system due to the fact that grain-grain collisions are inelastic. Like $\eta$ and $K, I$ depends on the collision rate, and hence on $\bar{v}$ and $s$. Simple models for these three quantities, as well as for the equation of state, are given in $\$ 5$.

\section{The microscopic model}

\subsection{The equation of state}

In order to proceed further, it is necessary to relate the pressure $p$, the transport coefficients $\eta$ and $K$, and the energy-sink term $I$ to the variables appearing in the various conservation equations. In order to determine these relationships, we make use of the simple picture provided by the cell model (Hirschfelder, Curtiss \& Bird 1964). The central grain is imagined to vibrate with average speed $\bar{v}$ in a random way so as to exert pressure $p$ on the surrounding grains.

A typical momentum transfer in a grain--grain collision is of the order of $m \bar{v}$. The collision time is

$$
\tau \sim s / \bar{v}
$$

+ Evidence that such a distribution obtains has been found by C. Campbell at Caltech (private communication) in Monte Carlo simulations of grain flow. See also Carlos \& Richardson (1968), who showed that the velocity distribution functions for tracer particles in a fluidized bed were approximately Maxwellian. 
Therefore the pressure exerted on the walls of the cell is

$$
p \sim \frac{m \bar{v}}{\tau} \frac{1}{d^{2}}
$$

since $s \ll d$ implies that the surface area of the cell is proportional to $d^{2}$. Collecting constant factors, this can be written as

$$
p=t d \rho \frac{\vec{v}^{2}}{s}
$$

where $t$ is a dimensionless constant, and $\rho \sim m / d^{3}$. As the grain temperature $\rho \vec{v}^{2}$ increases, the pressure rises, and conversely the pressure falls as the density decreases, as expected.

Equation (5.3) has the basic form of Van der Waal's equation of state, without the terms arising from the overlap of the attractive tails of the molecular potentials. This can be seen by rewriting $s$ in terms of the density, using (3.2), and then substituting into (5.3). The result is

$$
p\left(V-V_{0}\right) \approx \text { constant } N \times \frac{1}{2} m \bar{v}^{2},
$$

where $N$ is the number of grains and $V=N m / \rho$ is the volume. The quantity $V_{0}$ is like the usual excluded volume term, except that it is less than the corresponding quantity appearing in the standard Van der Waal's equation for dilute gases. The reason is that for close-packed systems each grain on the average excludes less volume than the same grain would in a dilute system. Although (5.4) is the more familiar, it turns out to be convenient to deal directly with (5.3).

Similar results for an equation of state have been derived by other workers, often with much more rigorous treatment of grain collision geometry and kinematics (Ogawa 1978). It is our intent here to establish the dependences of quantities like pressure and viscosity, and of the flow and temperature fields as well, upon the dynamic variables of interest. It is perhaps premature at this time to invest too much rigour in calculating exact numerical coefficients, when fundamental problems, such as choosing the correct boundary conditions, still remain unsolved.

\subsection{The coefficient of viscosity}

Here we wish to derive the dependence of $\eta$ upon the variables appearing in our description of the system. This is done in the simplest way by considering two adjacent 'layers' of grains. The gradient of the flow velocity $u$ is supposed to be in the $y$-direction perpendicular to these layers, so that on the average the upper layer moves with respect to the lower layer with relative velocity $\Delta u$. This is not meant to imply that motion necessarily occurs in these ordered assemblies, but is intended to focus attention on the difference in the mean velocities of neighbouring grains.

When grain collisions occur between the two layers, an average net momentum of magnitude $m \Delta u$ in the $x$-direction (direction of flow) is transferred. Since the collision rate is $\bar{v} / s$, the shear stress exerted by the upper layer, on the lower layer, in the $x$-direction, is

$$
\sigma \sim \frac{m \Delta u}{d^{2}} \frac{\bar{v}}{s}
$$

The increment $\Delta u$ is the change in $u$ over a distance of the order of $d$, so that $\Delta u / d \sim \mathrm{d} u / \mathrm{d} y$. Defining the stress as

$$
\sigma=q d^{2} \rho \frac{\bar{v}}{s} \frac{\mathrm{d} u}{\mathrm{~d} y}
$$


where $q$ is a dimensionless constant, and comparing this two-dimensional example with the viscous-stress term in (4.3), gives

$$
\eta=q d^{2} \rho \frac{\bar{v}}{s}
$$

as the expression for the viscosity coefficient.

The viscosity depends upon $\bar{v}$, the thermal velocity, which is the solution to the energy equation (4.4), and thereby the coupling between the momentum and energy equations is made explicit. In the case of free flow, where $\bar{v}$ must be scaled by $u$, it is clear that $\sigma$ will be nonlinear in $u$, as anticipated. This leads to the form of the stress law originally anticipated by Bagnold (1954), and derived again in similar form by other authors (e.g. McTigue 1978). However, it is clear from the development of (5.7) that, having gone as far as expressing $\eta$ in terms of the collision rate $\bar{v} / s$, it is now up to the equations themselves to supply self-consistently the precise form of the stress law, and we allow them to do so below.

\subsection{The coefficient of thermal diffusivity}

The thermal diffusivity coefficient $K$ appearing in (4.4) can be found in a similar way. The energy flux due to grain-grain collisions is the average net energy transfer per collision times the collision rate divided by the area. The mean energy transfer is $m \bar{v} \Delta \bar{v}$, where $\Delta \bar{v}$ is the difference in mean thermal velocity between neighbouring grains, and thus the energy flux is

or

$$
\begin{gathered}
Q \sim \frac{m \bar{v} \Delta \bar{v}}{d^{2}} \frac{\bar{v}}{s} \\
Q=-r d^{2} \frac{\bar{v}}{s} \frac{\mathrm{d}}{\mathrm{d} y}\left(\frac{1}{2} \rho \bar{v}^{2}\right),
\end{gathered}
$$

where $r$ is a dimensionless constant. The term in (4.4) involving $K$ is just the divergence of the internal energy flux, and thus we can identify

$$
K=r d^{2} \frac{\bar{v}}{s}
$$

Note that in (4.4) this term does not involve the flow velocity $u$ explicitly, and therefore in principle it is present even when there is no macroscopic motion of the granular system. It describes the transport of energy as 'heat'. Jenkins \& Cowin (1979) have described the necessity of allowing for the diffusion of 'heat', and they introduced an equation analogous to (5.9). In their model, however, $K$ was taken to be a constant. Here $K$, like $\eta$, remains to be determined by the equations themselves.

\subsection{The collisional energy sink}

The other term in (4.4) that is independent of $u$ is $I$, representing the energy irretrievably lost to the system due to the fact that grain-grain collisions are inelastic. Let $e$ be the coefficient of restitution describing the collision of two grains. The relative collision velocity is of the order of $\bar{v}$. Therefore, the loss of energy per collision is

$$
\Delta E \sim\left(1-e^{2}\right) \frac{1}{2} m \bar{v}^{2} .
$$

Multiplying by the collision rate $\bar{v} / s$ and the number density $n$ gives the rate at which energy is lost through collisions per unit volume per second:

$$
I=\gamma \rho \frac{\bar{v}^{3}}{s}
$$




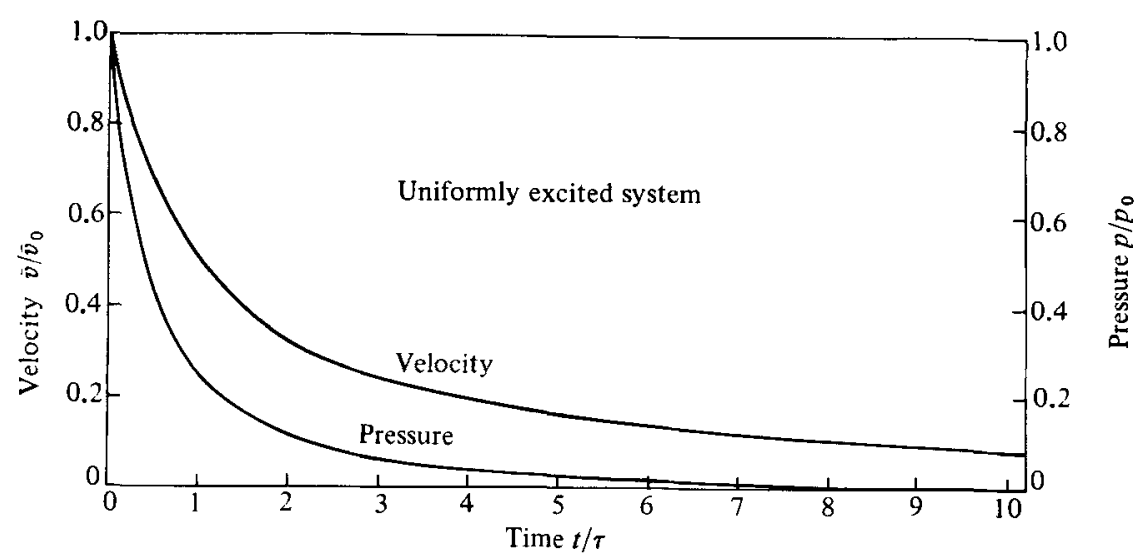

Figure 2. The thermal velocity $\bar{v}$ and the pressure $p$ are plotted versus elapsed time for a system initially excited in a spatially uniform way. $\tau=s_{0} / 2 \gamma \bar{v}_{0}$.

where $\gamma$ is a dimensionless factor proportional to $1-e^{2}$. The dissipation function has also been discussed by other authors (Ogawa 1978), but its importance has not been sufficiently emphasized. As shown below, a granular flow of even nearly perfectly elastic spheres, can deviate greatly from the flow of a similar fluid composed of exactly elastic spheres.

\section{Solutions of the equations of motion}

\subsection{Uniformly excited system}

One of the simplest problems to analyse involves a system at some initial uniform temperature, characterized by a thermal velocity $\bar{v}(0) \equiv \bar{v}_{0}$, which is then allowed to decay with time. There is no flow, so $u=0$ everywhere, and all spatial derivatives vanish. Then, from (4.4), we have

$$
\frac{\partial}{\partial t}\left(\frac{1}{2} \rho \bar{v}^{2}\right)=-\gamma \rho \frac{\bar{v}^{3}}{s}
$$

The solution, illustrated in figure 2 , is

$$
\bar{v}(t)=\frac{\bar{v}_{0}}{\left(2 \gamma \bar{v}_{0} t / s_{0}\right)+1},
$$

where $s_{0}$ is the (constant) mean grain separation (we take $g=0$ ). The time-scale for decay is

$$
\tau=\frac{s_{0}}{2 \gamma \bar{v}_{0}}
$$

so that if the coefficient of restitution $e$ is nearly unity, $\gamma \approx 0$, and the system takes a long time to cool. The very slow rate of damping $\left(\sim t^{-1}\right.$ at large $\left.t\right)$ is due to the face that as the mean energy per particle decreases, the time between collisions, $s_{0} / \bar{v}$, becomes increasingly long. According to (5.3), the pressure decreases, as shown in figure 2 , in the following manner:

where $p_{0}$ is the pressure at $t=0$.

$$
p(t)=\frac{p_{0}}{\left[\left(2 \gamma \bar{v}_{0} t / s_{0}\right)+1\right]^{2}}
$$

This example is interesting because it illustrates the point, which we wish to make 
now, that the thermal velocity $\bar{v}$ need not always scale with a flow velocity $u$. Here the scale is given by $\bar{v}_{0}$, and was introduced as an initial condition. In other problems of interest, certain boundary conditions on $\bar{v}$ will need to be introduced, and these boundary conditions provide a vehicle by which a new velocity scale may be introduced. This is another reason for constructing the viscosity in the self-consistent way described above, because the flexibility of that formulation allows automatically for a variety of scaling possibilities. If the shear stress term is forced by hand to be bilinear in $u$, as is usually done, then whole classes of solutions are summarily eliminated.

\subsection{Steady-state system with no flow}

Another interesting and simple problem which does not involve macroscopic flow is to determine the steady-state temperature and density of a granular system contained between two large parallel plates separated by a distance $h \gg d$. Because of the steady-state condition, all time derivates are set equal to zero. We assume there is no gravitational field $g$. The energy equation under these circumstances reduces to

$$
\boldsymbol{\nabla} \cdot\left(K \nabla \frac{1}{2} \rho \vec{v}^{2}\right)=I,
$$

which says that in the steady-state condition with no flow, the rate at which energy is conducted into a volume must just balance the rate at which energy is lost in that volume by collisions. If $x$ measures the perpendicular distance away from a confining wall located at $x=0$, then (6.5) becomes

$$
\frac{1}{2} r d^{2} \frac{\mathrm{d}}{\mathrm{d} x} \frac{\bar{v}}{s} \frac{\mathrm{d}}{\mathrm{d} x} \bar{v}^{2}=\frac{\gamma \bar{v}^{3}}{s} .
$$

By taking the interior derivative in this equation, it can be seen that the factor $\bar{v}^{2} / s$ appears on both the left-hand and right-hand sides. By $(5.3), \bar{v}^{2} / s$ is proportional to the pressure. Since $g$ has been taken to be zero, however, the momentum equation (4.3) implies that the pressure is a constant:

$$
p=p_{0} .
$$

Therefore (6.6) is actually a linear equation for $\bar{v}$,

$$
\frac{\mathrm{d}^{2} \bar{v}}{\mathrm{~d} x^{2}}=\frac{\gamma}{r d^{2}} \bar{v}
$$

and the solution is

$$
\bar{v}(x)=\bar{v}_{0} \mathrm{e}^{-x / \lambda},
$$

where $\bar{v}_{0}$ is a constant and the lengthscale is given by

$$
\lambda=\left(\frac{r}{\gamma}\right)^{\frac{1}{2}} d .
$$

In writing (6.9) we have assumed $h \gg \lambda$.

This result requires some discussion. The quantity $\vec{v}^{2} / s$ which was eliminated in favour of the pressure above recurs frequently in the examples worked out below. For zero-gravity problems with no flow the pressure will be constant, and therefore two powers of thermal velocity can always be eliminated whenever they occur, with a concomitant simplification of the energy equation. When the pressure is not constant, as is the case when an external force field is present, then $\bar{v}^{2} / s$ can still be eliminated, but instead of a constant $p_{0}$, a substitution involving a function of position, $p \rightarrow p(x)$, will be involved. We could rewrite the energy equation in terms 
of $p$ directly, but this would require specifying models for $K, \eta$ and $I$, and for the equation of state, at the very outset. We prefer to leave the equation in the more general form as written, and then to explore the consequences of a particular choice for the transport coefficients and equation of state. Other choices (corresponding to a dilute grain system with inelasticity, say) can then be made as desired.

In arriving at $(6.9)$ we have applied the boundary conditions $\bar{v}(h) \rightarrow 0$ and $\bar{v}(0) \rightarrow \bar{v}_{0}$. The boundary condition at $x=0$ corresponds to requiring that the mean random velocity $\bar{v}$ have a constant value at the wall, and therefore energy must be supplied there to balance the rate of energy absorption in collisions. In a true fluid, constant velocity $\bar{v}_{0}$ would correspond to maintaining the wall at a constant temperature. At the microscopic level, random vibrations of molecules at the surface of the wall supply energy to fluid molecules through collisions. The wall bounding the granular system, however, is in general not composed of other grains all vibrating at random and independently, so that it is difficult to produce a precise analog of the molecular energy transfer mechanism for the granular system. In writing down the solution (6.9), we are merely demanding, without regard to the problems that would face the experimentalist, that a certain random velocity $\bar{v}_{0}$ characterize grains very near the bounding surfaces. In attempting to actually produce this condition one might, for example, fix the wall roughness to have the same scale as a typical grain diameter $d$. The wall could then be driven by an appropriate shaking device, with mean amplitude on the order of or less than $d$, and with a suitably random frequency spectrum. A mixture of vertical as well as horizontal displacements, with respect to the wall boundary, would be desirable. Even with these refinements, the motion of each element of the wall is highly correlated with that of every other element, and therefore it may be difficult experimentally to insure that a random velocity field is produced.

In fact, in a standard shaker experiment with perpendicular wall motion, especially if the motion is large compared with a grain diameter, it is likely that coherent grain motion will be produced. This motion will carry momentum away from the wall in an organized manner, and corresponds to the generation of a sound wave, with the grains as the elementary entities supporting the wave. While wave propagation is certainly subsumed under the set of equations (4.1), (4.3) and (4.4), it is not what we are investigating in the present example. Care must therefore be exercised in the comparison of predictions involving boundary conditions on $\bar{v}$ with standard shaker experiments.

From (6.10) the effect of the energy loss in grain-grain collisions can be seen. The lengthscale over which the random thermal velocity falls from its value at the wall to $1 / \mathrm{e}$ of that value is a multiple of the grain diameter $d$. In order for the continuum picture to obtain, the condition $\lambda \gg d$ must be true: This requires that $r / \gamma \gg 1$, a condition independent of $\bar{v}_{0}$ as long as the coefficient of restitution is not velocity dependent. To order unity, this is equivalent to requiring that the coefficient of restitution $e$ be very nearly unity. Thus because of the exponential way in which the surface disturbance is damped in the interior of the system, only grains which are nearly elastic will rigorously satisfy the conditions of the continuum hypothesis. If the grains are only moderately elastic, or are quite inelastic, then it is not in general possible to carry through the present analysis unmodified. What would be required in these latter cases would be to treat this thin transition layer separately - as in the treatment of a boundary layer in fluid mechanics - and then to match boundary conditions between this layer and the rest of the system at a suitably chosen point away from the wall. In the remainder of the paper, however, we specialize to very, 




Figure 3. Illustration of the definition of the free-space parameter $\Delta h$ for a channel of width $h$.

but not totally, elastic particles. We note that this condition can be relaxed somewhat for shear flows, where the internal generation of thermal energy can occur over distances large compared to $\lambda$.

Finally, it is necessary to check that $s \ll d$. Again eliminating $\bar{v}(x)$ in favour of the (constant) pressure $p_{0}$, we have

and therefore we require

$$
s=\frac{\vec{v}_{0}^{2} t d \rho}{p_{0}} \mathrm{e}^{-2 x / \lambda}
$$

$$
\vec{v}_{0}^{2} \ll \frac{p_{0}}{t \rho} .
$$

Unfortunately this relation cannot yet be used, because the value of $p_{0}$ is unknown. In an elastic grain system, or in a molecular gas, it would be unnecessary to supply power to the wall in order to maintain a given temperature, but rather, the temperature, density (i.e. $s$ ) and pressure would be constant throughout the volume. The pressure would then be determined from a knowledge of $s=s_{0}$ and $\bar{v}=\bar{v}_{0}$. However, for an inelastic grain system, $s$ and $\bar{v}$ are not constant. In order to determine $p_{0}$ we need one further piece of information, the total amount of free volume available to accommodate grain motion.

Imagine that all the grains in the channel are pushed, as illustrated in figure 3, to the right into a state where each grain touches its neighbours. The gap which 
develops between the wall and the surface of the packed grains is denoted by $\Delta h$. If for the moment we denote by $\rho_{0}$ the constant density of packed grains, and by $\rho=\rho(x)$ the density of grains found above for the vibrating wall, then

$$
\int_{0}^{h} \rho(x) \mathrm{d} x=\int_{0}^{h-\Delta h} \rho_{0} \mathrm{~d} x
$$

Using (3.2), and setting $\rho_{0} \approx m / d^{3}$, as in (3.3), we have, if $s \ll d$ as is to be required,

$$
\Delta h=\frac{3}{d} \int_{0}^{h} s(x) \mathrm{d} x
$$

The quantity $\Delta h$ is a new parameter whose value it is necessary to know in order to completely specify the state of the system. For all problems of the kind discussed in this section, and in many other problems as well, the 'free-space' parameter $\Delta h$ must be supplied. Knowledge of $\Delta h$ is equivalent to knowledge of the number of grains per unit area in the channel.

Applying the relation given by $(6.14)$ to $(6.11)$ yields

$$
p_{0}=\frac{3}{2} t \rho \bar{v}_{0}^{2} \frac{\lambda}{\Delta h},
$$

so that the pressure is proportional to the energy density at the surface. Combining (6.15) and (6.12) then gives, as the condition that $s \ll d$ everywhere,

$$
\frac{\lambda}{\Delta h} \gg 1 \text {. }
$$

Equation (6.16) expresses the physical requirement that if the total distance over which grains are dispersed is much greater than the total 'free distance', then the grains must be close together. In this example there is no limitation to the values which $\bar{v}_{0}$ may assume.

The density parameter $s$ is written explicitly from (6.11) and (6.15) as

$$
s=\frac{2}{3} d \frac{\Delta h}{\lambda} \mathrm{e}^{-2 x / \lambda},
$$

so that the density profile is independent of the way in which the grains at the bounding wall are excited.

\subsection{Steady-state system with no flow in gravitational field}

Here we imagine a bounding surface at $x=0$, perpendicular to the gravitational field $\boldsymbol{g}$, as illustrated in figure 4. A layer of grains of thickness $h_{0}, h_{0} \gg d$, is put on the surface, and the floor is then vibrated in such a way that the grains near the boundary have thermal velocity $\bar{v}_{0}$. The thickness of the layer then increases from $h_{0}$ to some height $h, \dagger$ whose value will depend upon $\bar{v}_{0}$. Arguments similar to those used to derive (6.14) lead to the equation

$$
h=h_{0}+\frac{3}{d} \int_{0}^{h} s(x) \mathrm{d} x,
$$

which will be found useful for the complete specification of the solution.

+ This is one case where the importance of coordination number and packing geometry is critical for a realistic analysis, since a (moderate) vibration applied to loosely-packed grains will cause the final height $h$ to be less than $h_{0}$. Here we must imagine starting from a close packed configuration. 


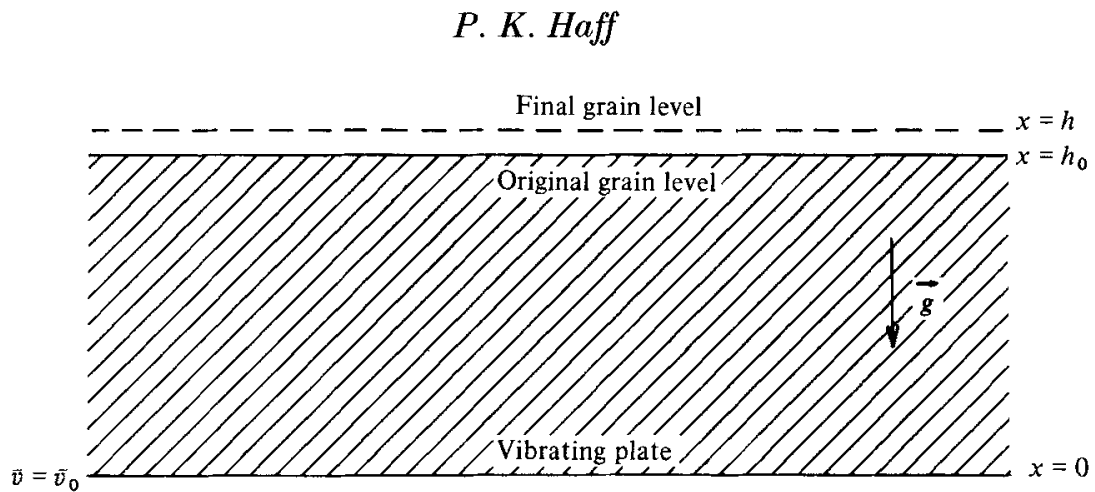

Figure 4. Illustration of the expansion of an initially packed configuration, in a gravitational field, upon supplying energy at the bottom plate $(x=0)$. The free surface rises from $x=h_{0}$ to $x=h$.

According to the momentum equation (4.3), the pressure at any point in the fluid is

$$
p(x)=\rho g(h-x)+p_{0} \equiv \rho g\left(h^{\prime}-x\right),
$$

where $p_{0}$ is the applied pressure at the top surface and

$$
h^{\prime} \equiv h+p_{0} / \rho g \text {. }
$$

For a free surface $p_{0}=0$, but for the moment it will be convenient to carry along the extra constant term.

The energy equation reduces to $(6.5)$, but since $p$ is no longer constant, the substitution for $\tilde{v}^{2} / s$ leads to a result different from $(6.8)$, namely

$$
\frac{\mathrm{d}^{2} \bar{v}}{\mathrm{~d} z^{2}}+\frac{1}{z} \frac{\mathrm{d} \bar{v}}{\mathrm{~d} z}-\bar{v}=0
$$

where $z$ is a dimensionless variable given by

$$
z=\frac{h^{\prime}-x}{\lambda}
$$

The solutions to (6.21) are the modified Bessel functions of zero order, $I_{0}(z)$ and $K_{0}(z)$.

Let us try the boundary condition $\bar{v}(0)=\bar{v}_{0}$, as before, but, at the upper surface, let the energy flux $Q$ vanish (5.9), i.e set

$$
\left.K \frac{\mathrm{d}}{\mathrm{d} x} \bar{v}^{2}(x)\right|_{h}=0 .
$$

This would be the usual condition at a free surface. Taking the derivative and rewriting in terms of the pressure gives

$$
\left[p(x) \frac{\mathrm{d} \bar{v}}{\mathrm{~d} x}\right]_{h}=0 .
$$

If $p_{0}=0$, then $(6.24)$ is evidently satisfied automatically since $p(h)$ would be zero. This corresponds to $\bar{v}^{2}(h) / s(h)=0$. In this case $x \rightarrow h$ corresponds to $z \rightarrow 0$. As the function $K_{0}(z)$ is singular here, $\bar{v}(x)$ must be given by $I_{0}$ alone. But because $I_{0}$ does not vanish at $z=0, \bar{v}(h)$ is non-zero. The only way in which $p(h)$ can vanish then is if $s(h) \rightarrow \infty$. This clearly violates the condition $s \ll d$. The basic problem we have encountered is the breakdown of the cell model at a free surface. Any impulse, however slight, in the upward direction, will allow a particle to move off unhindered 
to infinity. There is a physical analogue to this situation which is actually encountered under certain vigorous flow conditions in chutes, where jostling of grains at the surface leads to the development of a thin upper layer of particles which are dispersed many grain diameters apart from each other. This phenomena, which may be termed gravitational saltation, is made possible by the accessibility of free space at an open surface.

In fact, the upper layer of grains does not move off to infinity because it is restrained by the gravitational force; a particle receiving an upward impulse at the surface moves on a ballistic trajectory and shortly returns at some other point. There is no room in the model we have presented so far to account for these gravitational effects. Gravity is included in the momentum equation (4.3) and the energy equation (4.4), but these equations refer to momentum and energy attributes of 'fluid particles ' which themselves contain many grains. They do not refer to momentum and energy properties of individual grains. Thus, the equation of state (5.3) and the various transport relationships developed earlier do not contain any dependence on $g$. This is a good approximation so long as the grain trajectory between collisions is essentially a straight line. This will be true if the grain kinetic energy is much greater than the change in gravitational potential energy experienced over a distance $s$, which is the mean distance between collisions, i.e. if

$$
\frac{\bar{v}^{2}}{g s} \gg 1
$$

In terms of the pressure this can be written as

$$
\frac{p}{\rho g d} \gg 1
$$

which says that $p$ must substantially exceed the force required to lift a grain through its own diameter. The expression appearing on the left-hand side of (6.25) is a microscopic version of the Froude number. The Froude number itself is of the form $F=u^{2} / g l$, where $u$ is a typical flow velocity and $l$ a characteristic macroscopic length in the fluid system. If $F \gg 1$, then gravitational effects are generally not of major importance. The value of the microseopic Froude number, which we may represent by $f=\bar{v}^{2} / g s$, tells whether the effects of $g$ must be included in the description of a system at the level of the individual grain. In most fluid systems, $f \gg 1$, because thermal velocities are high. $\dagger$ However, in the example of this section, the requirement that $s \rightarrow \infty$ in order that the pressure may vanish at $x=h$ leads to $f \leqslant 1$. Thus, if we wish to specify in detail the behaviour of the granular system near its free surface, we would need to modify the equation of state, the thermal diffusivity, and so on. This generalization would presumably allow for a consistent accounting of the effects of grains on ballistic (i.e. curved) trajectories at the surface.

Generally, $f$ will be small only in a thin layer at the surface. Below this region, $f \gg 1$, and (5.3) is valid. We may therefore think of gravitational effects as producing a kind of boundary layer at the free surface, which may be called the saltation layer. Since a different equation of state characterizes this part of the system, it can be thought of as essentially a different fluid from that comprised by the more dense material below. Our approach then will be to treat the material which comprises the bulk of the system, between $x=0$ and the bottom of the saltation layer, according to (5.3), and to take account of the existence of the saltation layer through appropriate boundary conditions.

$\dagger$ Note that $f \gg 1$ and $F \leqslant 1$ can be mutually consistent conditions. 
For a grain layer $h^{\prime}$ that is sufficiently thick, a modest input of energy at $x=0$ will produce no significant thermal vibration near the surface. If we imagine that $h<h^{\prime}$ is the height below which saltation effects can be ignored, and if we denote by $p_{0}$ the pressure at $h$, then

$$
h=h^{\prime}-\frac{p_{0}}{\rho g},
$$

from (6.20). If $\bar{v}(h) \approx 0$ here (and the energy flux $Q \approx 0$ also), then, because $I_{0}$ is an exponentially increasing function of its argument for large $z\left(I_{0}(z) \sim(2 \pi z)^{-\frac{1}{2}} \mathrm{e}^{z}\right.$ as $z \rightarrow \infty$ ), we can write

$$
\bar{v}(x) \approx \bar{v}_{0} \frac{I_{0}[(h-x) / \lambda]}{I_{0}(h / \lambda)}
$$

where $\bar{v}_{0}$ is the velocity maintained at $x=0$, and, since we take $h \gg \lambda, \bar{v}(h) \approx 0$. In this case, however, we might as well write $I_{0}$ in its asymptotic form to get

$$
\bar{v}(x) \approx \bar{v}_{0}\left(\frac{h^{\prime}}{h^{\prime}-x}\right)^{\frac{1}{2}} \mathrm{e}^{-x / \lambda}
$$

which is similar to the earlier result (6.9) obtained in the absence of gravity. This result will apply up to some height which is less than $h^{\prime}$, the true top of the layer. For modest energy input at $x=0$, however, $h^{\prime}$ and $h$ will be practically coincident, at least to within a few grain diameters. Thus for practical purposes, when the externally applied excitation is not too large, (6.29) will be valid over the entire volume, except within a few grain diameters of the surface.

The density function $s$ is given by

$$
s=\frac{t d h^{\prime} \vec{v}_{0}^{2} \mathrm{e}^{-2 x / \lambda}}{g\left(h^{\prime}-x\right)^{2}},
$$

so that the density is smallest at $x=0$, and increases toward the surface. If $\lambda \ll h$, then $s$ essentially goes to zero before the top surface is reached. If $p_{0}$ is taken equal to zero, and $h^{\prime} \rightarrow h$, then the 'saltation' spike at $x=h$ is clearly seen in (6.30).

The height $h$ of the layer, for a given value of $\bar{v}_{0}$, and for $h^{\prime} \approx h_{0}$, is found from (6.18) to be

$$
h-h_{0} \approx \frac{3}{2} t \frac{v_{0}^{2}}{g h_{0}} \lambda .
$$

The expansion $h-h_{0}$ increases with the temperature $\frac{1}{2} m \vec{v}_{0}^{2}$ at the bottom of the layer, but scales inversely with the strength of the gravitational attraction. A thinner initial layer $h_{0}$ will expand, for a given $\bar{v}_{0}$, more than a thicker $h_{0}$, because in the latter case the increased weight of the overburden serves to compress the fluid near $x=0$, where most of the expansion occurs.

Finally, we need to check under what conditions $s \ll d$ is true. From $(6.30)$, evaluated sufficiently far from the free surface, this may be stated as

$$
\frac{\vec{v}_{0}^{2}}{g h_{0}} \ll 1 \text {. }
$$

which says that the wall vibration must be much less than that vibration which could be capable of tossing a grain to a height $h_{0}$, or, for a given $\bar{v}_{0}$, that either gravity or the thickness of the layer must be great enough to keep the grains near the bottom under sufficient pressure that they cannot move far apart from one another. This condition can be expressed another way by combining (6.31) and (6.32); i.e., in order 




Figure 5. Illustration of Couette flow geometry. We look for solutions which depend on the transverse coordinate $y$ only. The free-space parameter is $\Delta h$, as in the static example of figure 3 .

that $s \ll d$, the absolute linear expansion $h-h_{0}$ must be small compared with the scale length imposed by the inelasticity of the grain collisions:

$$
h-h_{0} \ll \lambda \text {. }
$$

\subsection{Steady-state Couette flow with no gravity}

In this subsection we consider a grain system enclosed by two parallel plates which move with constant velocity $U$ with respect to each other. We look for steady-state solutions. The plate separation $h$ is constant, and to be definite we take the lower plate to be at rest. Figure 5 illustrates the coordinate system; the $y$-axis is perpendicular to the plates, while the $x$-axis is parallel to them. The system is of infinite extent in the $x$ - and $z$-directions. We search for solutions of the equation of motion that depend only on $y$.

Since $\eta=\eta(y)$, the $y$-component of the momentum equation gives $\mathrm{d} p / \mathrm{d} y=0$ so that the pressure in the channel is constant:

$$
p=p_{0}=t d \rho \frac{\vec{v}^{2}}{s}
$$

In a similar way, an examination of the $x$-component of (4.3) shows that the shear stress is a constant throughout the system:

$$
\sigma=\sigma_{0}=\eta \frac{\mathrm{d} u}{\mathrm{~d} y}
$$

The energy equation reduces to

$$
0=\frac{\partial}{\partial x_{k}}\left[u_{i} \eta\left(\frac{\partial u_{i}}{\partial x_{k}}+\frac{\partial u_{k}}{\partial x_{i}}\right)+K \frac{\partial}{\partial x_{k}} \frac{1}{2} \rho \bar{v}^{2}\right]-I
$$

As illustrated in the previous no-flow examples, the pressure expression (6.34) can be used to eliminate powers of $\bar{v}$ in (6.36). We also find that the shear-stress relation (6.35) is useful in eliminating explicit reference to the flow velocity $u$ in (6.36). Thus by incorporating $(6.34)$ and $(6.35)$ into $(6.36)$ we have

$$
\frac{\mathrm{d}^{2} \bar{v}}{\mathrm{~d} y^{2}}+\omega^{2} \bar{v}=0
$$


where

$$
\omega^{2}=\frac{t^{2} \sigma_{0}^{2}}{r q d^{2} p_{0}^{2}}-\frac{\gamma}{r d^{2}}
$$

The frequency $\omega$ is constant, but its value is not yet known, because the ratio $\sigma_{0} / p_{0}$ remains unknown. Equation (6.37) is therefore an eigenvalue equation.

Boundary conditions are taken as follows. For the flow velocity $u(y)$, which is always in the $x$-direction, we simply demand that

$$
\begin{aligned}
& u(0)=0, \\
& u(h)=U .
\end{aligned}
$$

These equations reflect the usual fluid-mechanical requirements that the fluid at a surface follow the motion of that surface. For a granular system, it is possible that slippage occur at a bounding plane, in which case the grain flow velocity would be different from the wall velocity. However, for a sufficiently rough surface, which we postulate here, (6.39) and (6.40) should be correct.

The boundary conditions on $\bar{v}$ are harder to specify with confidence. A complete solution of the motion would replace an outright specification of $\bar{v}$ at the boundary with some criterion involving the nature of the grain-wall interaction. Such a criterion is not presently at hand. Nevertheless, it will be recognized that there is utility in the examination of solutions to the flow equations even if somewhat arbitrary boundary conditions must be invoked. In fluid mechanics the number of analytically soluable problems is so small that nearly any such solution is of some interest. In granular mechanies the number of analytical solutions is even smaller. Thus exact solutions of kinetic theories of grain flow are felt to be worth the price of some uncertainty as to the realism of selected boundary conditions.

Here, we set $\bar{v}$ equal to zero (with one exception, as discussed below). This has the advantage that such boundary conditions are relatively easy to work with, and that one can imagine constructing a wall so that grain vibration there is minimized (Jenkins \& Cowin 1979). (The wall should be 'soft' in order to make the coefficient of restitution for a grain collision with the wall small.) One could equally well choose $\bar{v}$ to have non-zero boundary values, but such a choice is more tedious to work with, and would provide no new qualitative understanding.

We note from (6.38) that evidently $\omega^{2}$ can be either positive or negative. Assume it is negative: then the curvature of $\bar{v}$ within the channel is positive (the solutions are exponentials). Since $\bar{v}$ is an intrinsically non-negative quantity, we cannot require that $\bar{v}$ vanish at both $x=0$ and $x=h$, unless $\bar{v} \equiv 0$ everywhere. For $\omega^{2}<0$, then, let us try

$$
\begin{aligned}
& \bar{v}(0)=\bar{v}_{0}, \\
& \bar{v}(h)=0 .
\end{aligned}
$$

This situation corresponds to vibration of the lower stationary plate only. The upper plate is imagined to be 'soft' so that grain-plate collisions are effectively damped.

Since the solution decreases monotonically between $y=0$ and $y=h, \mathrm{~d} \bar{v} / \mathrm{d} y$ is negative everywhere. The energy flux is $Q=-K \rho \bar{v}(\mathrm{~d} \bar{v} / \mathrm{d} y)$. Since $K>0$, we have $Q>0$, and therefore energy flows from $y=0$ toward $y=h$, just as heat would flow from a region of high temperature to a region of low temperature. The source of the energy is the externally applied vibration of the lower plate.

In the limit $v \ll \bar{v}_{0}$ and $\omega h \gg 1$ (to be checked later on), we find

$$
\bar{v}(y)=\bar{v}_{0} \mathrm{e}^{-\omega y}
$$


which satisfies (6.37) and, approximately, the boundary conditions (6.41) and (6.42). The flow-velocity gradient is related to $\bar{v}$ by

$$
\frac{\mathrm{d} u}{\mathrm{~d} y}=\frac{\sigma_{0} t}{q d p_{0}} \bar{v}
$$

and the integration of this equation, together with (6.38), gives

$$
\omega \approx \frac{1}{\lambda}
$$

and thus

$$
\frac{\sigma_{0}}{p_{0}}=\frac{q}{t} \frac{d}{\lambda} \frac{U}{\bar{v}_{0}}
$$

The condition $w h \gg 1$ thus becomes $h \gg \lambda$, which is usually satisfied easily, and we get the thermal velocity explicitly

$$
\bar{v}(y)=\bar{v}_{0} \mathrm{e}^{-y / \lambda},
$$

which is identical to the result obtained in the static examples considered above. By going to the limit $U \ll \bar{v}_{0}$ we have retained only the effect of wall vibration on $\bar{v}$; the next-higher-order term in $U / \bar{v}_{0}$ would begin to show the effect of the flow pattern itself upon the thermal velocity field.

The pressure and shear stress are calculated to be

$$
\begin{gathered}
p_{0}=\frac{3}{2} t \rho \bar{v}_{0}^{2} \frac{\lambda}{\Delta h}, \\
\sigma_{0}=\frac{3}{2} q \rho \bar{v}_{0} U \frac{d}{\Delta h} .
\end{gathered}
$$

The pressure $p_{0}$ is independent of the flow velocity, for small $U$, and is determined by the energy flux at the lower wall. The stress $\sigma_{0}$ is directly proportional to the speed $U$ at which the upper plate moves. Equations (6.48) and (6.49) are thus analogous to the hydrodynamical solutions for Couette flow. This is a reasonable result because in ordinary hydrodynamical systems the flow velocity is much less than the molecular thermal velocity. We can see in this example the advantage of treating the momentum and energy equations on an equal footing. If in the momentum equation the shear stress term had been forced by hand to be quadratic in $U$, then $\sigma$ would have scaled with $U^{2}$, i.e. it would not have been possible to go to the hydrodynamical limit.

Finally, the actual flow pattern of course is not hydrodynamical, because of the importance of grain inelasticity:

$$
u(y)=U\left(1-\mathrm{e}^{-y / \lambda}\right) .
$$

Since the scale distance $\lambda$ is much less than the channel width $h$, the region of fluidization is confined to a thin layer of thickness $\approx \lambda$ near the lower plate. Most of the matter in the channel moves as an essentially non-deforming plug.

We turn now to the case of high flow rates. If the shear stress $\sigma$ is large enough, it is not unreasonable to expect that enough thermal energy would be generated internally in the grain system by friction to require conduction to the plates. Since the internal energy flux, as discussed above, is proportional to $\mathrm{d} \vec{v} / \mathrm{d} y$, a negative curvature for $\bar{v}$ is required in order for heat to flow out of the channel. This 


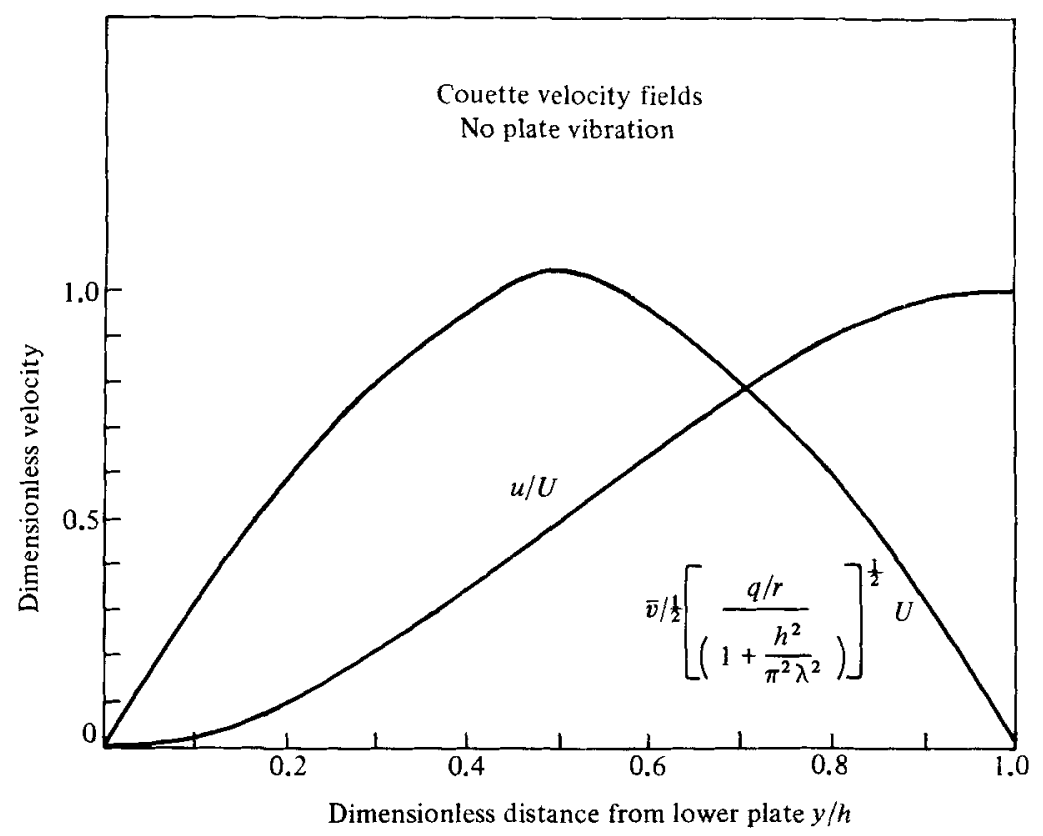

Figure 6. The thermal and flow velocities for the Couette flow example illustrated in figure 5 are plotted versus distance from the lower plate. The thermal velocity $\bar{v}$ is set equal to zero at each plate.

corresponds to $\omega^{2}>0$ in $(6.37)$, i.e. to oscillatory solutions. To be definite, the boundary conditions on $\bar{v}$ can be taken to be

$$
\bar{v}(0)=\bar{v}(h)=0,
$$

although this choice is not essential.

The thermal velocity $\tilde{v}$ has the solution

where $a$ is a constant and

$$
\bar{v}=a \sin \omega y,
$$

$$
\omega=\pi / h
$$

The pressure $p_{0}$ and shear stress $\sigma_{0}$ are constant throughout the flow volume. Integrating (6.44) to obtain the flow velocity and applying the boundary conditions in conjunction with the free-space relation (6.18) give the following explicit expressions for the thermal and the flow velocities:

$$
\begin{gathered}
\bar{v}(y)=\frac{1}{2}\left(\frac{q}{r}\right)^{\frac{1}{2}} \frac{1}{\left(1+h^{2} / \pi^{2} \lambda^{2}\right)^{\frac{1}{2}}} U \sin \frac{\pi y}{h}, \\
u(y)=\frac{1}{2} U\left(1-\cos \frac{\pi y}{h}\right) .
\end{gathered}
$$

The velocity $U$ of the upper plate is the only velocity in the problem, and therefore it scales both $\bar{v}$ and $u$, as anticipated. Figure 6 illustrates these two velocity fields. The conduction of friction-generated energy from the interior of the flow to the plates is clearly demonstrated. For modest values of $\bar{v}$ at the plates, the same general kinds of solutions could be obtained. 
Formulae for the pressure and stress are obtained in the course of solving for $\bar{v}$ and $u$; we find that the pressure is given by

and the stress by

$$
p_{0}=\frac{3}{8} \rho U^{2} t \frac{q}{r} \frac{1}{1+h^{2} / \pi^{2} \lambda^{2}} \frac{h}{\Delta h}
$$

$$
\sigma_{0}=\frac{3}{8} \pi \rho U^{2} \frac{q^{\frac{3}{2}}}{r^{\frac{1}{2}}} \frac{1}{\left(1+h^{2} / \pi^{2} \lambda^{2}\right)^{\frac{1}{2}}} \frac{d}{\Delta h} .
$$

In (6.56) and (6.57), for the first time, the expected quadratic dependence on velocity appears.

Note that knowing the shear stress and pressure we can calculate the ratio of frictional to collisional energy loss rates,

$$
\frac{\dot{\mathscr{E}}_{\mathrm{f}}}{I}=\frac{t^{2}}{\gamma q} \frac{\sigma_{0}^{2}}{p_{0}^{2}}
$$

Since $\sigma_{0}$ and $p_{0}$ are constants, the loss-rate ratio is the same everywhere in the system. Recalling the expression $(6.38)$ for $\omega^{2}$, we see that this quantity can be written in terms of $\dot{\mathscr{E}}_{\mathrm{f}} / I$ as

$$
\omega^{2}=\frac{1}{\lambda^{2}}\left(\frac{\dot{E}_{\mathrm{f}}}{I}-1\right)
$$

If, within a given volume, the collisional loss of energy exceeds the frictional loss, $\omega^{2}$ is negative, and there must be a net flux of internal energy via conduction into the interior of the channel in order to sustain steady-state motion. These are the positive-curvature solutions considered earlier. If the frictional loss should exceed the collisional loss, then an excess of internal energy is generated per unit volume, and net conduction toward the walls must occur. These solutions are the ones of negative curvature.

Finally we need to check under what circumstances the condition $s \ll d$ is satisfied. The density parameter $s$ is given explicitly as

$$
s=\frac{2}{3} \frac{d}{h} \Delta h \sin ^{2} \frac{\pi y}{h}
$$

Therefore the present description will be valid if

$$
\Delta h \ll h \text {. }
$$

This condition is easier to satisfy than the relation $\Delta h \ll \lambda$ which characterized the solutions for the case $\bar{v}_{0} \gg U$. In the latter example, strong plate vibration generates high porosity and low density in a very limited region, so that it is easy to force $s$ to exceed $d$ in the vicinity of the plate. However, according to $(6.60)$, the decrease in density for high flow rates $\left(U \gg \bar{v}_{\mathrm{o}}\right)$ is spread much more uniformly over the channel region, and therefore a much larger value of the free space parameter $\Delta h$ is tolerable.

\subsection{Plug formation in Couette flow}

There is always the possibility that at certain places within the channel grains may become so closely packed that effects of Coulomb friction (which we have not treated fully) dominate over collisional energy loss. In this case, there may arise regions where grain separation goes to zero, so that a 'plug' forms. Indeed, in the limit $s \ll d$ that we have been discussing, one may argue that, for many or most materials, plug formation is likely. The limit of closely packed grains moving without plug formation, is, of course, a very useful limit to explore, and we have done so in this paper. 
Nevertheless, it is worthwhile to consider what the present model has to say about the existence of plugs.

Imagine that a group of grains becomes joined together in a thin layer along the upper plate, so that the region between $y=y_{0}$ and $y=h$ is composed of grains in the state $s=0$. The grains do not move with respect to the plate $y=h$, and therefore the situation is as if the remainder of the granular fluid were being forced to flow between plates separated by a distance $y_{0}$ instead of $h$. If the plug were stable, the solutions for the velocities $U$ and $\bar{v}$, and for the forces $\sigma_{0}$ and $p_{0}$, could obviously be transcribed so that they applied to this new channel of decreased width.

Whether the plug is stable or not depends upon whether the forces deriving from the interlocked grains are strong enough to resist the shearing force $\sigma_{0}$ associated with the grain flow. The precise stability criterion is not known, but, to be definite, let us assume that if $\sigma_{0}$ is less than a 'critical' shear stress

$$
\Sigma_{0}=\mu p_{0}
$$

then the plug is stable. Here $\mu$ is a dimensionless constant which depends on the properties of the plug (not calculable in the present model) and $p_{0}$ is just the internal pressure. $\Sigma_{0}$ need not have this form; it might be taken simply to have a constant value. In any case, a criterion such as

$$
\sigma_{0}=\Sigma_{0}
$$

provides the extra condition needed in order to determine the value of $y_{0}$.

There is still an indeterminancy in the problem, however, because the plug could form at the lower plate $(y=0)$ instead of the upper plate. The value of $y_{0}$ calculated from (6.63) just tells us the width of the flow zone, but there is no way to predict where that zone will lie. It could lie in the middle of the channel, and be bordered both above and below by plugs.

There is yet a more complicated possibility. In (6.53) we have solved the eigenvalue equation for the thermal velocity $\bar{v}$ by requiring that $\omega=\pi / h$. However, if zones of plug formation are allowed, there is no reason why eigenvalue conditions like $\omega_{n}=$ $n \pi / y_{n}$ might not be valid, where $y_{n}$ is the width of a particular flow zone, and $n$ is an integer. Thus, under appropriate conditions, a number of alternating flow zones separated by rigid (but generally moving) plugs may appear. Again there is a certain amount of indeterminancy associated with the number and position of these zones. If they exist, we can discuss their stability, but it is not possible to predict where within the channel they will lie.

Although these considerations are interesting, and may have significant effects in many realistic systems, we limit ourselves here to a discussion of systems for which closely packed grains exhibit no additional shear strength beyond that due to collisions.

\subsection{Steady-state Couette flow in a gravitational field}

Here we consider the same problem addressed in $\$ 6.4$, except that the gravitational field $\boldsymbol{g}$ is present. For simplicity, $\boldsymbol{g}$ is taken to be perpendicular to the confining plates. In the usual way the pressure is found to be

$$
p(y)=\rho g(h-y)+p_{0},
$$

where $p_{0}$ is the 'extra' (constant) pressure which will be generated by the relative motion of the plates. The shear stress $\sigma_{0}=\eta \mathrm{d} u / \mathrm{d} y$ is again constant, and substitution into the energy equation for derivatives of the flow velocity $u$ gives the equation for 
thermal velocity $\bar{v}$. Performing a similar substitution for the pressure, which is not constant, in order to eliminate higher powers of $\bar{v}$, leads to the equation

where

$$
\frac{\mathrm{d}^{2} \bar{v}}{\mathrm{~d} z^{2}}+\frac{1}{z} \frac{\mathrm{d} \bar{v}}{\mathrm{~d} z}-\left(1+\frac{\nu^{2}}{z^{2}}\right) \bar{v}=0
$$

The length $h^{\prime}$ is greater than the width of the channel:

$$
h^{\prime}=h+p_{0} / \rho g \text {. }
$$

Equation (6.65) has solutions which are modified Bessel functions of purely imaginary order $I_{v}$, and $K_{v}$, with $\nu$ given by (6.66). However, because the solutions cannot be expressed as a finite combination of elementary functions, which solutions are the main object of this paper, we turn our attention to the simpler problem of perfectly elastic grains undergoing Couette flow in a gravitational field. In this limit (6.65) can be written as

$$
\frac{\mathrm{d}^{2} \bar{v}}{\mathrm{~d} z^{2}}+\frac{1}{z} \frac{\mathrm{d} \bar{v}}{\mathrm{~d} z}+\frac{c^{2}}{z^{2}} \bar{v}=0
$$

where

$$
c=|\nu|=\frac{1}{(r q)^{\frac{1}{2}}} \frac{t \sigma_{0}}{\rho g d},
$$

and, redefining the variable $z$,

$$
z=\frac{h^{\prime}-y}{d}
$$

The solutions to (6.69), with boundary conditions given by $(6.39),(6.40)$ and $(6.51)$, are

$$
\begin{aligned}
& \bar{v}=\frac{1}{2}\left(\frac{q}{r}\right)^{\frac{1}{2}} U \sin \left(c \ln \frac{z}{z_{0}}\right), \\
& u=\frac{1}{2} U\left(1+\cos \left(c \ln \frac{z}{z_{0}}\right)\right),
\end{aligned}
$$

while the constant components of the pressure and stress are given by

$$
\begin{gathered}
p_{0}=\frac{\rho g h}{\mathrm{e}^{\pi / c}-1}, \\
\sigma_{0}=\frac{3 \pi}{8} \frac{q^{\frac{3}{2}}}{r^{\frac{1}{2}}} \rho U^{2} \frac{d}{\Delta h} .
\end{gathered}
$$

The constant $z_{0}$ is given by

$$
z_{0}=\frac{p_{0}}{\rho g d}
$$

Note that neither $\bar{v}$ nor $u$ are linear functions of the plate velocity $U$. This is not the case when gravity is absent: according to (6.54) and (6.55) $\bar{v}$ and $u$ are simply proportional to $U$ when $\boldsymbol{g}=0$. In the presence of a gravitational acceleration, however, an extra time dimension is introduced into the problem, and it can be compensated for only by the appearance of extra factors of $U$. 


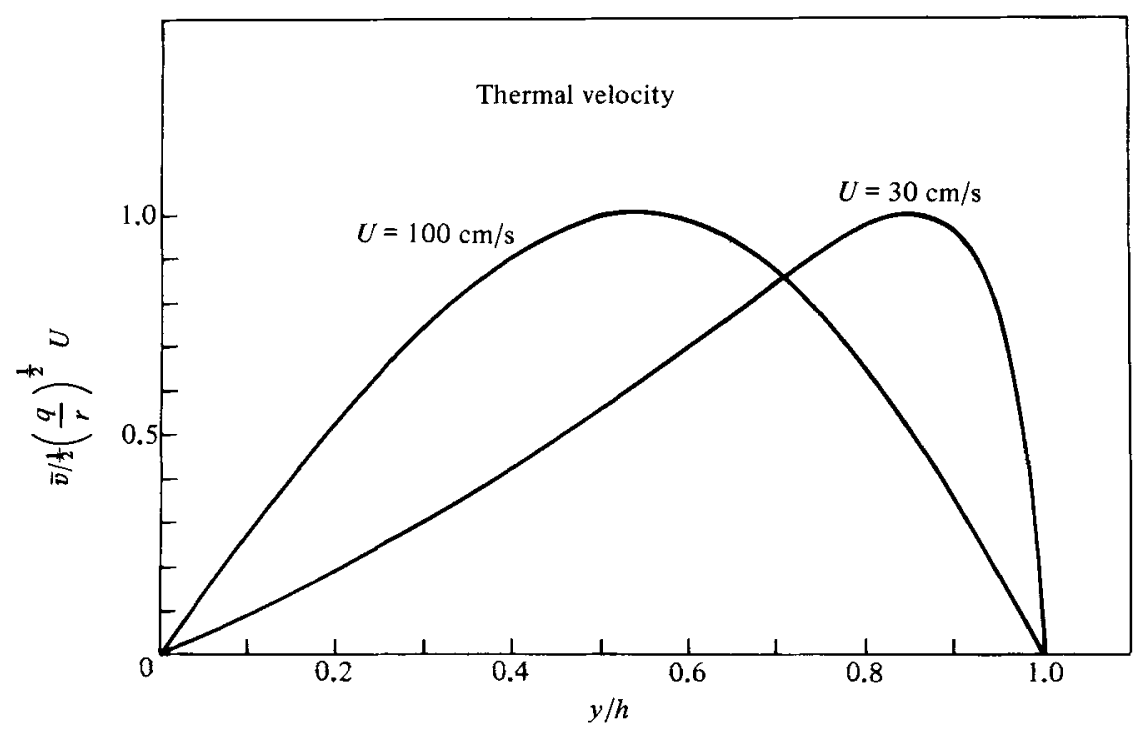

Figure 7. Illustration of the thermal velocity $\bar{v}$ for Couette flow in a gravitational field, for two different velocities $U$ of the upper plate. At the higher plate velocity, $U=100 \mathrm{~cm} / \mathrm{s}$, the shape of the thermal velocity curve resembles that found in the absence of a gravitational field (figure 6). At lower plate velocities, the fluidization is most pronounced near the upper plate. In these calculations $\Delta h=1 \mathrm{~cm}, g=1000 \mathrm{~cm} / \mathrm{s}^{2}$ and $q=r=t=1$.

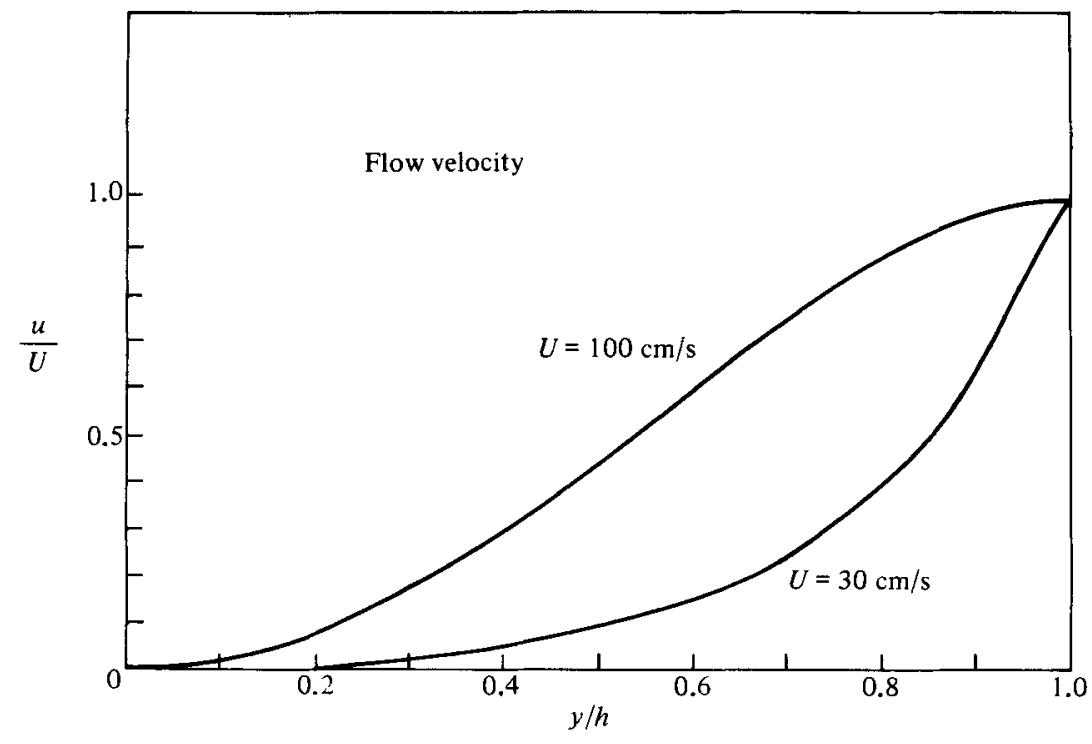

FIgURE 8. Illustration of the flow velocity $U$ for Couette flow in a gravitational field, for two different velocities $U$ of the upper plate. At the higher plate velocity, $U=100 \mathrm{~cm} / \mathrm{s}$, the shape of the flow velocity curve resembles that found in the absence of a gravitational field (figure 6). At lower plate velocities, most of the shearing occurs in the upper part of the channel. In these calculations $\Delta h=1 \mathrm{~cm}, g=1000 \mathrm{~cm} / \mathrm{s}^{2}$ and $q=r=t=1$.

Figures 7 and 8 illustrate the behaviour of $\bar{v}$ and $u$ respectively, for two different values of the plate velocity $U$. The main effect of the gravitational field $g$ is to shift the principal region of thermalization from the centre of the channel toward the upper plate, and thereby to cause the principal zone of shearing to lie nearer the top plate than the bottom one. 


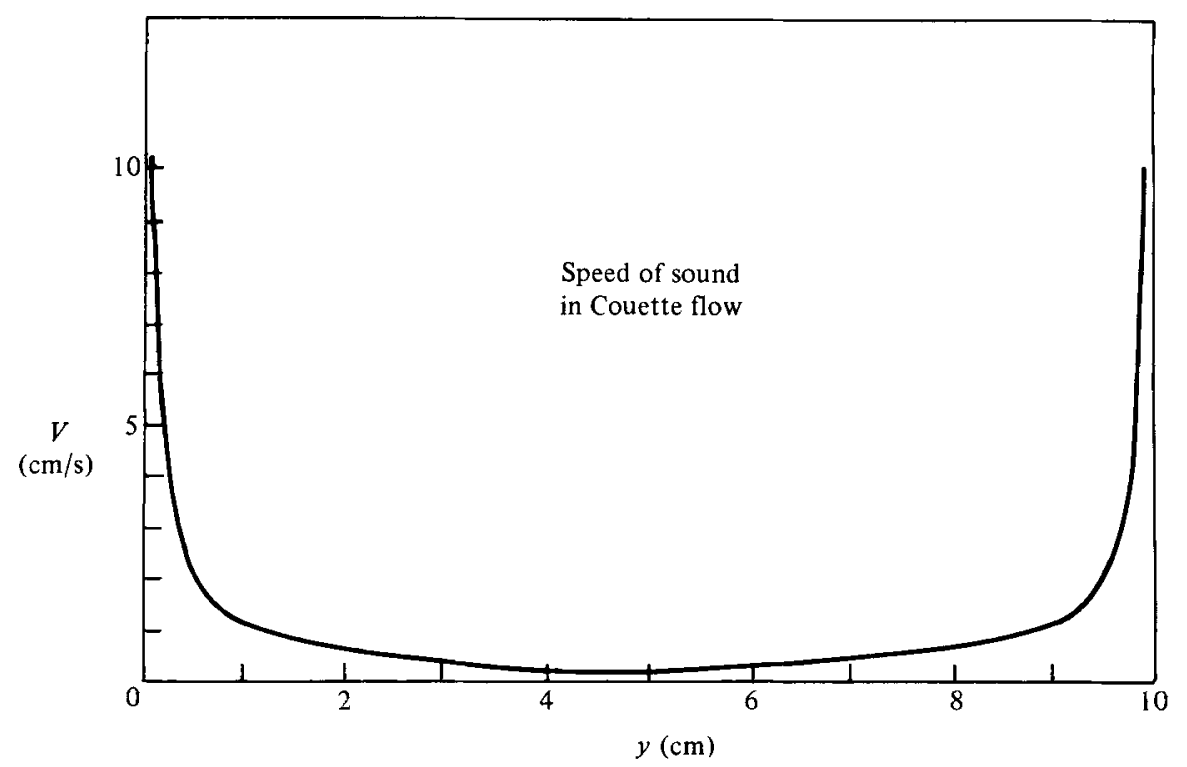

Figure 9. The speed of sound is plotted versus position in the channel for zero-gravity Couette flow characterized by the following parameters $U=10 \mathrm{~cm} / \mathrm{s}, h=10 \mathrm{~cm}, \Delta h=1 \mathrm{~cm}, \gamma=0.5$, $d=0.1 \mathrm{~cm}$ and $q=r=t=1$.

\section{Supersonic flow}

The speed of sound $V$ in flowing granular material can be estimated from the expression

$$
V \approx \bar{v} \frac{d}{s}
$$

$V$ is much larger than $\bar{v}$ because most of the path length of the sound wave is within the grain itself, and the speed of sound within a single grain can be taken to be infinite for our purposes. By taking characteristic values of $\bar{v}, d$ and $s$ in a Couette flow geometry, one can show that at the centre of the channel $V$ exceeds the flow velocity, for elastic grains, by about an order of magnitude. But for realistic inelastic grains, $V$ is slightly less than the flow velocity. Thus we come to the important conclusion that kinetic grain flow is intrinsically a supersonic phenomenon.

This conclusion is true if $\bar{v}$ vanishes or is small at the boundaries. If the grain system is externally fluidized by vibration of the walls, or if the walls are good insulators (in the granular sense), then the value of $\bar{v}$ both at the walls and in the channel interior will increase, while the density $s$ remains unchanged, so that $V$ could exceed $u$ everywhere.

Actually, from (7.1) we can obtain an idea of the magnitude of the speed of sound not only at the centre of the channel, but everywhere between the plates. Substituting for $s$ and $\bar{v}$ from (6.54) and (6.60) respectively gives

$$
V=\frac{3 \pi}{4} \frac{\lambda}{\Delta h} \frac{U}{\sin (\pi y / h)},
$$

if $\gamma$ is not too small. Thus the speed of sound is smallest at the centre of the channel, and according to (7.2) becomes large without limit at the walls. The supersonic region is accordingly confined to a limited zone within, but not extending to, the edges of 
the channel. Of course, at the edges, $V$ does not actually diverge, but approaches the velocity with which sound would be transmitted through a network of mutually touching grains.

The extent of the zone over which the flow is supersonic is determined by the relation

$$
u(y)>V(y)
$$

where $u$ is given by (6.55) in the present example. This can be simplified to the condition

$$
\sin \frac{\pi y}{h}\left(1-\cos \frac{\pi y}{h}\right)>\frac{3}{2} \pi \frac{\lambda}{\Delta h}
$$

which does not involve the plate velocity $U$. For elastic grains, $\lambda \rightarrow \infty$, and the flow is always subsonic, no matter how fast the wall moves. However, for grains with a realistic coefficient of restitution, the condition (7.4) is often satisfied over some region since $\lambda / \Delta h$ is usually a small quantity. The speed of sound is plotted in figure 9 for the case $\gamma=0.5$.

These results mean that a small disturbance created in the central region of the channel under conditions of steady flow will propagate only in the downstream direction. Perturbations applied in the subsonic boundary layers, however, will be able to propagate in both directions. Finally, the placement of obstacles in the flow channel will result in the generation of shock waves.

\section{Conclusion}

The above developments represent an attempt at a systematic description of one type of grain flow, where relatively elastic grains are very close to, but in general not touching, other neighbouring grains. A crucial step in arriving at a self-consistent description is the explicit inclusion of the energy equation. This involves introduction of the variable $\bar{v}$, the 'thermal' velocity. In solving various problems, somewhat arbitrary boundary conditions on $\bar{v}$ have been assigned, and it remains for the future to decide how these conditions will be applied in realistic cases. For experiments involving energy supply to the granular medium through the boundaries, it is as yet unclear how closely the coherent vibration of container walls will simulate the picture of a random excitation of near-boundary grains as described in the preceding sections.

This paper has been restricted principally to a consideration of analytic solutions to certain static and steady-state problems. This has usually meant, especially in the case of non-zero flow fields, that it was possible to solve only for elastic grains in a gravitational field, or for inelastic grains without gravity. Since all laboratory experiments must involve inelastic grains and since most experiments must also be performed in a gravitational field, it is not generally possible to compare the present calculations with the result of flow experiments, even if the uncertainties over the proper boundary conditions are resolved.

In spite of these imperfections, it is felt that the results of the present paper represent a positive step in the understanding of grain flow under certain rheological conditions. Thus the critical importance of including grain inelasticity even for a qualitative description becomes evident. And the prediction that grain flow is supersonic may lead to new ways of thinking about standard grain-flow problems.

There are many areas in which further investigation is required. These include the theory of sound propagation and damping in a fluidized system (since energy influx 
at a vibrating boundary may be best described in terms of sound waves), and the treatment of gravitational flows with a free surface. A difficulty encountered in attempting to treat this latter flow pattern is that, as in the example of $\S 6.3$, a non-zero 'thermal' energy at the free surface leads to a saltation-like effect ('gravitational saltation'). Here, in a thin layer, the density goes rapidly to zero, and this surface boundary layer must be treated on a separate footing from the bulk of the flowing material.

In addition to remedying specific problems of the particular model presented here, one also has a general desire to improve the fundamental basis of the model, which at present is entirely heuristic, by appeal to a more detailed microscopic approach. Further developments remain for the future, but it is hoped that the present work, in spite of its limitations, will serve as a useful point of departure for more detailed investigations of granular flow.

Many of the results of this paper sprang from discussions with C. E. Brennen, S. E. Koonin, R. Shreve, T. A. Tombrello and C. C. Watson. Partial support was provided by the National Science Foundation (PHY79-23638).

\section{REFERENCES}

ACk ERmann, N. L. \& Shen, H. T. 1978 Flow of granular material as a two-component system. In Cowin \& Satake (1978), pp. 258-265.

Ackerman, , N. L. \& Shen, H. 1982 Stresses in rapidly sheared fluid-solid mixtures. J. Engng Mech. Div. ASCE 108 (EM1), 95-113.

Bagnold, R. A. 1954 Experiments on a gravity-free dispersion of large solid spheres in a Newtonian fluid under shear. Proc. R. Soc. Lond. A225, 49-63.

Bagnold, R. A. 1956 The flow of cohesionless grains in fluids. Proc. R. Soc. Lond. A249, $235-297$.

Campbell, C.S. \& Brennen, C. E. 1982 Computer simulations of chute flows of granular materials. In Deformation and Failure of Granular Materials (ed. P. A. Vermeer \& H. J. Luger), pp. 515-521. A. A. Bulkenna, Rotterdam.

Carlos, C. R. \& Richardson, J. F. 1968 Solids movement in liquid fluidised beds - I. Particle velocity distribution. Chem. Engng Sci. 23, 813-824.

Cowin, S. C. 1978 Microstructural continuum models for granular materials. In Cowin \& Satake (1978), pp. 162-170.

Cowrn, S. C. \& M. Satake (eds.) 1978 Proc. US-Japan Seminar on Continuum-Mechanical and Statistical Approaches in the Mechanics of Granular Materials. Gakujutsu Bunken Fukvukai. Tokyo, Japan.

Hirschfelder, J. O., Curtiss, D. F. \& Bird, R. F. 1964 Molecular Theory of Gases and Liquids. Wiley.

Jenkins, J. T. \& Cowin, S. C. 1979 Theories for flowing granular materials. In Mechanics Applien to the Transport of Bulk Materials (ed. S. Cowin). ASME AMD-31, pp. 79-89.

Jenkins, J. T. \& SAVAGE, S. B. 1981 The mean stress resulting from interparticle collisions in a rapid granular shear flow. In Continuum Models of Discrete Systems 4 (ed. O. Brulin \& R. K. T. Hsieh), pp. 365-371. North-Holland.

McTigue, D. F. 1978 A model for stresses in shear flow of granular material. In Cowin \& Satake (1978), pp. 266-271.

MARBLe, F. E. 1964 Mechanism of particle collision in the one-dimensional dynamies of gas-particle mixtures. Phys. Fluids 7, 1270-1282.

OGAwA, S. 1978 Multitemperature theory of granular materials. In Cowin \& Satake (1978), pp. 208-217.

Ogawa, S., Umemura, A. \& Oshima, N. 1980 On the equations of fully fluidized granular materials Z. angew. Math. Phys. 31, 483-493. 
Osнrma, N. 1978 Continuum model of fluidized granular media. In Cowin \& Satake (1978), pp. 189-207.

SAVAGE, S. B. 1979 Gravity flow of cohesionless granular materials in chutes and channels. J. Fluid Mech. 92, 53-96.

Savage, S. B. \& JefFrey, J. D. 1981 The stress tensor in a granular flow at high shear rates. J. Fluid Mech. 110, 255-272.

Shen, H. \& ACkermann, N. L. 1982 Constitutive relationships for fluid-solid mixtures. J. Engng Mech. Div. ASCE 108 (EM5), 748-763. 